\title{
Ethnobotanical Studies on the Use of Medicinal Plants among Forest Fringe Communities around the Kasewe Forest in Moyamba District, Southern Sierra Leone
}

\author{
James S. Eisah¹, Fallah Nyumah², Jonathan Johnny¹, Joseph F. Charles ${ }^{3}$ \\ ${ }^{1}$ School of Natural Resources Management, Njala University, Njala and Bo, Sierra Leone \\ ${ }^{2}$ Department of Environmental Science, William VS Tubman University, Harper, Liberia \\ ${ }^{3}$ Department of Interdisciplinary Research, Honors College of Research and Gender Studies, University of Liberia, Monrovia, \\ Liberia \\ Email: fallahnyumah@gmail.com
}

How to cite this paper: Eisah, J.S., Nyumah, F., Johnny, J. and Charles, J.F. (2021) Ethnobotanical Studies on the Use of Medicinal Plants among Forest Fringe Communities around the Kasewe Forest in Moyamba District, Southern Sierra Leone. American Journal of Plant Sciences, 12, 1963-1989.

https://doi.org/10.4236/ajps.2021.1212135

Received: August 1, 2021

Accepted: December 27, 2021

Published: December 30, 2021

Copyright $\odot 2021$ by author(s) and Scientific Research Publishing Inc. This work is licensed under the Creative Commons Attribution International License (CC BY 4.0).

http://creativecommons.org/licenses/by/4.0/

\section{(c) (i) Open Access}

\begin{abstract}
Forests are home to many flora and fauna species. Forest flora have been very important to man and sustainability of forest ecosystem. Forest flora provides provisional, regulatory, protective and cultural services. These services have been the foundation of civilization and development. Local communities depend on these natural resources for livelihood generation and cultural services. Local communities have been using medicinal plants to cure different ailments. In this study, an ethnobotanical survey was conducted to document medicinal plants diversity and use in forest fringe communities. The study adopted a method used by Martin in 1995 on Ethnobotanical reviews of medicinal plants. Ethnobotanical information was gathered through structured questionnaires administered to 57 inhabitants constituting key informants, community leaders, and household heads who are believed to have vast indigenous knowledge of medicinal plants. The snowball technique was used to identify respondents in communities around the Kasewe forest. For the diversity of medicinal plants use and associated indigenous knowledge, a plot of size $25 \mathrm{~m} \times 25 \mathrm{~m}$ was demarcated with a linear tape and ranging poles in the different habitat types in the study area. Sampling was stratified based on the size of habitat types. A total of 10 sample plots comprising of 4 plots in the closed forest; 3 plots in disturbed areas, 2 plots in farmland, and 1 plot in the grassland were studied. A total of 3377 individual plant stems were docu-
\end{abstract}


mented comprising of 84 individual species belonging to 53 families and 78 genera. Of the 84 individual plant species, 42 were medicinal. The most dominant family was Malvaceae. Tree species were the most dominant life form representing $32.14 \%$ followed by shrubs 30.5 , herbs $26.8 \%$ and climbers $10 \%$. Majority of the plant species are of little conservation concern and few have been categorized as endangered and vulnerable that need special conservation attention. Species with the highest density was Chromolaena odorata $110 \mathrm{~m}^{2}$, while Xylopia quintais $0.1 \mathrm{~m}^{2}$ had the least density per hector. The Shannon-wiener index recorded 1.236 as the highest in plot 3 while plot 10 in the grassland was considered as the lowest with 0.757 . The finding revealed that 92.98\% of respondents around the Kasewe forest in the Moyamba district used medicinal plants to treat therapeutic ailments. At the same time, a very limited proportion depends on other medication sources to treat therapeutic ailments. $98.25 \%$ of the respondents, mostly older people, know medicinal plants to treat therapeutic ailments. All parts of the plant were indicated to be used for treatment of different ailments. However, the root (43.8\%), bark $(36.8 \%)$ and leaves $(35 \%)$ are the most common plant parts used to treat different therapeutic ailments. $96.49 \%$ of the respondents have knowledge on administering prepared herbal medicine.

\section{Keywords}

Ethnobotanical, Medicinal Plants, Forest Fringe Community

\section{Introduction}

Medicinal Plants in Sierra Leone and other parts of the world play viable role in maintaining biodiversity and healthy ecosystems and livelihood of inhabitants of forest-fringe communities. Medicinal Plants offer range of ecosystem services and economic benefits, including provisioning, regulating, aesthetic, and spiritual values [1]. Medicinal plants in the forest ecosystem of Sierra Leone are beneficial to local people for a diverse range of benefits. They serve as a source of therapeutic ailments across all cultures, traditions, and spiritual rites of all the ethnic groups in the country, with specificity to the Sande and Poro societies widely practiced by the mendes, kissi and other ethnic groups in the country [2]. The use of plants as medicines is not only peculiar to Sierra Leone but represents, by far, the most significant human use of the natural resources in the world with more than 50,000 species reported to be of medicinal value. Medicinal plants are used by residents of forest fringe communities for livelihood generation, treatment for therapeutic ailment, cultural and spiritual norms [1] [2]. Over the years, medicinal plants have frequently been the primary source of drugs for preventing and treating different kind of diseases among local people and manufacturing conventional drugs use by modern health centers [2]. The use of plants as medicines to treat illness among rural people has a long history. Several plant 
species in Sierra Leone are of medicinal value to the citizens in the country, especially at the Kasewe forest. [1] investigated that medicinal plants such as Garcinal kola, Nauclea Diderrichii, Ceiba Pantandra, Ipomoea Involculture are used by the inhabitants of forest fringe communities and traditional herbalist for allopathic medicine for the treatment of common sickness. Findings from [3] also noted that medicinal Plant has been a regular source of substances for treating various diseases, especially in rural areas. Many studies [1] [2] [3] [4] have also discovered and documented more than 200 medicinal plant species used to cure more than 100 diseases and illnesses in Sierra Leone.

According to [5], 85\% of the medicine used for primary healthcare is derived from plants, which indicates that the chemical and genetic constituents of these plants are increasingly explored by pharmaceutical companies for profit and human benefit. Worldwide, there is an increasing interest in medicinal plants' use due to the growing public awareness of medicinal plants pharmacologically active compounds. In Canada, about $70 \%$ of the population used medicinal plants as therapeutic aliments for the citizens [6]. Another finding in England indicated that $47 \%$ of the population used medicinal plant for disease treatments [7].

The Kasewe forest is a storehouse of precious medicinal plants and other species that are economically viable. The Kasewe Forest is one of the single most essential storehouses of terrestrial biological diversity in Sierra Leone. Inhabitants around the Kasewe forest highly depend on the natural ecosystem and resources for livelihood generation, such as traditional herbal medicine for the treatment of both human and livestock diseases and source of protein from wildlife [1] [2]. More recently, [8] broadly defined ethnobotanical as human evaluation and manipulation of plant materials, substances, and phenomena in societies, and [9], related it as the study of how people make use of plants. However, [3] described ethnobotany as the entire realm of the beneficial relationship between Plant and man. These definitions emphasize a connection between people of a given community or society, the environment, and the plant diversity in that particular community. The importance of indigenous knowledge is overwhelming. [10] valued it as the sum of the experience that forms the basis for decision making for familiar and unfamiliar problems and challenges in a local community. [11] indigenous knowledge represents an immense valuable database that provides mankind with an insight into how numerous forest fringe communities have interacted with the changing environment, providing local solutions for local problems and suitable ways for coping with challenges posed by specific conditions. They provide a wide range of amenities to people residing around the forest and more beneficial to forest fringe communities. Medicinal plants play a vital role in supporting human primary health care to forest fringe communities proximate to a forested region globally, especially developing countries like Sierra Leone where the country is still struggling to improve its health care system for rural people. Medicinal plants in a forest ecosystem and woody flora help support many other organisms and have created the ambience for multifa- 
ceted mechanisms to maintain high levels of genetic and biological diversity in a forest ecosystem and promote livelihood sustenance for rural people. Like in the Himalayas, medicinal plants are harvested in large magnitudes in some regions by large amounts of people, for whom this action of collecting medicinal Plant from forests provides a considerable share of their income [12]. The livelihood remunerations of medicinal plants trade have been studied in Nepal, where an estimated 323,000 - 470,000 households (2.6 million people) are engaged in collecting wild medicinal plants for sale. The over-dependence on traditional herbal medicine results from unaffordability and lack of modern restorative services around the Kasewe forest reserve [1] [2]. The natural ecosystem and genetic resources are heavily depleted due to increased population, lack of awareness, technical know-how, and poor regulation of herbalists in Sierra Leone. To maintain these communities' ecosystem health, research to quantify medicinal plants diversity and uses is needed. Detailed ethnobotanical information on medicinal plants in the Kasewe forest is also needed, as noted by [1] [2]. Due to the aforementioned challenges and inaccessibility of contemporary health facilities, especially in Sierra Leone, and most people are still forced to use medicinal plants for their common day ailments, there is a high need to document traditional knowledge and uses of Plant for posterity. It was from this backdrop this research was conducted to determine local people's interactions with medicinal plants and document current medicinal in the Kasewe Forest Community from this background. Therefore, the study aimed to assess medicinal plant species used to manage human ailments and indigenous knowledge.

\section{Materials and Methods}

\subsection{Description of Study Area}

Kasewe forest is located in hills along the Freetown-Bo highway in the Moyamba District, south of Sierra Leone. It was designated as a forest reserve in 1914 and contains semi-deciduous and evergreen forests covering $1224 \mathrm{~km}^{2}$ (2306.8 ha). It is made up of volcanic rocks, and the hills stand about $500 \mathrm{~m}$ above the country's interior plains [13]. The study area is gradually deforested with some open patches due to anthropogenic activities carried out by inhabitants of these communities for livelihood sustenance (Figure 1).

\subsection{Data Collection Technique}

The data used were primary and secondary. Secondary data were obtained from related internet journal sources. The study adopted a method used by [14] on Ethnobotanical reviews of medicinal plants. The primary data was collected using survey and field measurements. Structured questionnaires were administered to selected categories of people who are believed to have vast indigenous knowledge of medicinal plants. The snowball technique was used to identify respondents in communities around the Kasewe forest. Questionnaires were asked on demographic, kind of Plant used, part and ailment treated. 


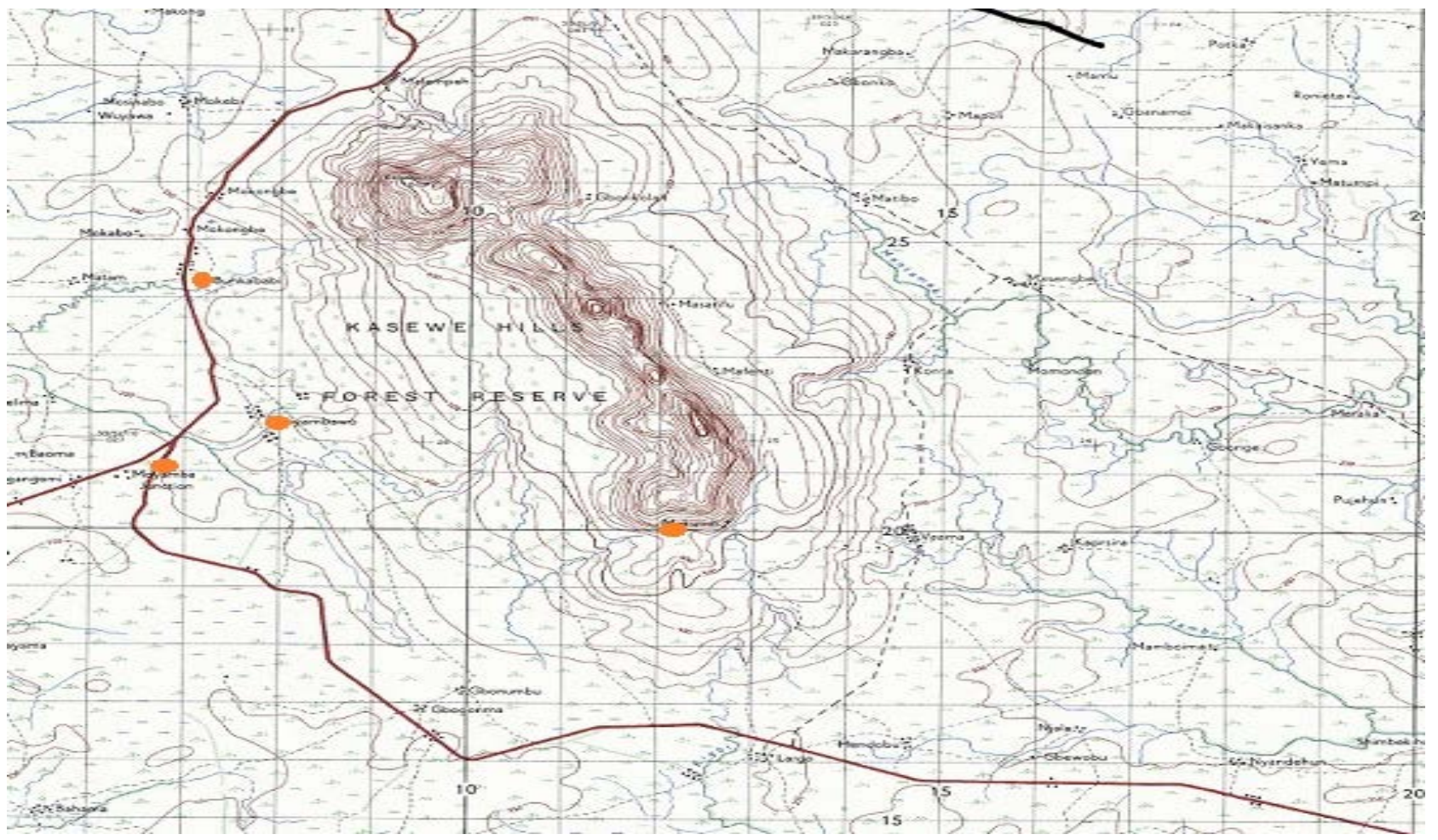

Figure 1. Map of the study area.

\subsection{Plant Diversity Studies on Medicinal Plants}

A Sample plot of size $25 \mathrm{~m} \times 25 \mathrm{~m}$ was demarcated with a linear tape and ranging poles in the different habitat types in the study area. Sampling was stratified based on the size of habitat types. A total of 10 sample plots were created during the plant diversity studies at the Kasewe forest reserve (4 plots in the closed forest; 3 plots in disturbed areas, 2 plots in farmland around the Kasewe forest; and 1 plot in the grassland). All plants, including trees, shrubs, climbers, and herbs, were identified within each plot. Plant identification was achieved in the field with the assistance of an experienced taxonomist, two forest guards and six persons were selected from the forest fringe communities and reserve for the identification of medicinal plants. Species that were difficult to identify in the field were compared with already identified specimens at the herbarium to confirm the field plant identification.

\subsection{Sampling Technique}

Respondents were sampled randomly from six forest fringe communities around the Kasewe forest reserve. A total of 57 well-informed respondents, including herbalists, community leaders, and household heads, were interviewed. The sampling technique was gender-sensitive, and both genders participated in the study (Table 1).

\subsection{Data Analysis}

Data from ethnobotanical studies of medicinal plant species were analyzed using 
Table 1. Number of communities around the Kasewe forest interviewed.

\begin{tabular}{ccc}
\hline No & Community Name & Number of Persons Interviewed \\
\hline 1 & Bongorla & 10 \\
2 & Bonkababay & 14 \\
3 & Koilu Moyambawo & 9 \\
4 & Vaama Kasewe & 9 \\
5 & Moyambawo & 8 \\
6 & Mor sheriff & 7 \\
Total & & $\mathbf{5 7}$ \\
\hline
\end{tabular}

Microsoft Excel, and the results obtained were presented in graphs, pie charts, and tabular forms as appropriate.

\subsubsection{Calculation of Ecological Indices of Plant Species}

Density, relative density, frequency, percentage frequency, relative frequency, and species diversity were calculated using the formulae given below by [15]:

1) Density $=\frac{\text { Total Number of individuals of a plant species in all sampled plots }}{\text { Total number of sampled plots }}$ Relative density of a species A(\%)

2) $=\frac{\text { Total Number of individual of Species }}{\text { Total number of individuals of all species }} \times 100$ Frequency of a species 3) $=\frac{\text { Number of quadrats sampled in which a species occurred }}{\text { Total number of quadrats sampled }}$

4) Relative frequency of species $A=\frac{\text { Number of the frequency of species }}{\text { The total frequency of all species }} \times 100$

5) Frequency $(\%)=\frac{\text { number of quadrants in which a species occurred }}{\text { Total number of quadrats sampled }} \times 100$

\subsubsection{Species Distribution Patterns}

The distribution of individuals of a population may be one of three patterns: random, uniform, and aggregate [10]. According to [16], individuals are randomly distributed when the variance mean ratio is 1 but aggregated when the ratio is greater than 1 . On the other hand, when the ratio is less than 1, individuals are uniform. The distribution patterns of the species sampled within the study sites were classified into mainly random and aggregated patterns, and the analysis was conducted using Biodiversity pro.

\subsubsection{Species Diversity}

A diversity index is the measure of species diversity in a given community. Diversity was measured by recording the species' number (species richness) and their relative abundance in the different plots. 


\subsubsection{Shannon Diversity Index}

Shannon-Weaver diversity index $(H)$ is a commonly used diversity index that considers both the abundance and evenness of species present in the community. It is explained by the formula below [17]. The species diversity and richness of the forest were calculated using Simpson's and Shannon Wiener's diversity index. Shannon Wiener diversity index is given as:

$$
\text { Shannon } \operatorname{Index}(H)=-\sum_{i=1}^{s} p_{i} \ln p_{i}
$$

The Shannon index is an information statistic index, which means it assumes all species are represented in a sample and randomly sampled. In the Shannon index, $p$ is the proportion $(n / N)$ of individuals of one particular species found (n) divided by the total number of individuals found $(N), \ln$ is the natural $\log , \Sigma$ is the sum of the calculations, and $s$ is the number of species.

\section{Results and Discussions}

\subsection{Social Demographic Information of Respondents}

Fifty-seven (57) respondents were interviewed in six communities around the Kasewe forest in Moyamba district. Most of the respondents were above 50 representing $38.56 \%$. This finding indicates that elderly people have more knowledge on medicinal plants than the younger generation. Similar finding was reported [18] where he found that majority of traditional knowledge and ethnobotanical information can be retrieved from the elderly population who are engaged into medicinal use. This implies that, the passing away of these elderly practitioners connotes the loss of this precious traditional knowledge particularly in cases where such wealth of knowledge has not been passed on to other younger generations. [19] also found that older people knew more about medicinal uses for native plants than younger people, but there was no difference in knowledge levels about non-native plants. Females constituted the majority (54\%) of the people interviewed. Women around the Kasewe Forest are more involved in the use of medicinal plants as compared to men. Other studies have also accentuated that female are more involve in using medicinal plants for the treatment of different therapeutic ailments especially in rural areas [20] [21]. [20] reported that "women" in Katsina State, Nigeria have been using medicinal plants to cure various ailments associated with maternal health since time immemorial. This finding may help support women involvement in medicinal plants and the idea that medicinal plant knowledge increases over time.

\subsection{Floristic Composition of Species}

The total number of individual Plant stems counted was 3377, comprising 84 unique species from different vegetative zones comprising 52 families belonging to 78 genera. Out of these 81 unique species, 42 species were classified as medicinal plants and used by inhabitants of the forest fringe communities around the Kasewe forest in Sierra Leone. These species were classified to treat different therapeutic ailments and are listed in (Table 2). The life forms of composition 
Table 2. Plants encountered in the Kasewe forest.

\begin{tabular}{|c|c|c|c|}
\hline Scientific name & Family Name & Life Form & Conservation status \\
\hline Adenia lobata & Passiforaceae & Climber & Unknown \\
\hline Aframomum $s p p$ & Zingiberaceae & Herb & Unknown \\
\hline Allium ascalonicum & Amaryllidaceae & Herb & Unknown \\
\hline Alchornea cordifolia & Euphorbiaceae & Shrub & Least concern \\
\hline Albizia zygia & Leguminosaceae & Tree & Not Threatened \\
\hline Anisophyllea laurina & Rhizophoracea & Tree & Unknown \\
\hline Ananas Cosmosus & Bromeliaceae & Herb & Unknown \\
\hline Beilschmiedia Mannii & Lauracea & Shrub & Unknown \\
\hline Capsicum annuum & Solanacea & Herb & Least concern \\
\hline Cassia siberrianna & Fabaceae & Shrub & Least concern \\
\hline Caraca papaya & Meliaceae & Tree & Least concern \\
\hline Carapa Procera & Meliaceae & Tree & Least concern \\
\hline Cercestis afzelii & Araceae & Climber & Unknown \\
\hline Ceiba Pantandra & Bombacaceae & Tree & Least concern \\
\hline Chlorophoro regia & Moraceae & Tree & Unknown \\
\hline Chromolaena Odorata & Asteraceae & Herb & Unknown \\
\hline Citrus aurantifolia & Rutaceae & Tree & Unknown \\
\hline Clerodendron scandens & Verbenaceae & Climber & Unknown \\
\hline Costus afer & Zingiberaceae & Herb & Unknown \\
\hline Corchorous olitorius & Malvaceae & Herb & Unknown \\
\hline Desmodium adscendens & Papilionaceae & Herb & Least concern \\
\hline Dialium guineense & Fabaceae & Tree & Unknown \\
\hline Diodia scandens & Rubiaceae & Herb & Unknown \\
\hline Diospyros heudelotii & Ebenacea & Shrub & Unknown \\
\hline Fiscus exasperata & Moracea & Shrub & Unknown \\
\hline Garcinia Kola & Clusiacea & Tree & Vulnerable \\
\hline Gmelina Arborea & Verbenaceae & Tree & Least concern \\
\hline Gosspium spp. & Malvacea & Herb & Unknown \\
\hline Guibourtia Copalifera & Fabaceae & Tree & Unknown \\
\hline Harungana madagascariensis & Hypericaceae & Shrub & Least concern \\
\hline Hibiscus esculentus & Malvaceae & Herb & Unknown \\
\hline Hibiscus physaloides & Malvaceae & Shrub & Unknown \\
\hline Hippocratea Iotricha & Celastracea & Climber & Unknown \\
\hline
\end{tabular}




\section{Continued}

\begin{tabular}{|c|c|c|c|}
\hline Hymenocardia Acida & Euphorbiaceae & Shrub & Least concern \\
\hline Impoea involucrate & Convolvulaceae & Herb & Unknown \\
\hline Lantana camara & Verbenaceae & Herb & Unknown \\
\hline Lannea & Anacardiacea & Tree & Least concern \\
\hline Mareya Micrantha & Euphorbiaceae & Shrub & Least concern \\
\hline Mangifera indica & Anacardiaceae & Tree & Data Deficient \\
\hline Manihot esculenta & Euphorbiaceae & Herb & Data Deficient \\
\hline Mimosa pudica & Fabaceae & Herb & Least concern \\
\hline Morinda germinata & Rubiaceae & Shrub & Unknown \\
\hline Musanga cecropiodes & Moraceae & Tree & Unknown \\
\hline Musa paradisiaca & Musaceae & Tree & Unknown \\
\hline Musa sapientum & Musaceae & Tree & Unknown \\
\hline Nesogordnia papaverifera & Malvacea & Tree & Vulnerable \\
\hline Newboudia laevis & Bignoniaceae & Shrub & Unknown \\
\hline Nuclea diderrichii & Rubiaceae & Shrub & Unknown \\
\hline Nuclea latifolia & Rubiaceae & Shrub & Unknown \\
\hline Ochthocosmus africnas & Ixonanthaceae & Shrub & Unknown \\
\hline Ocimum viride & Labiatae & Herb & Unknown \\
\hline Ouratea flava & Ochnacea & Tree & Unknown \\
\hline Oryza sativa & Gramineae & Herb & Least concern \\
\hline Panicum afzelli & Gramineae & Herb & Unknown \\
\hline Parinari excelsa & Chrysobalanaceae & Tree & Least concern \\
\hline Paullinia pinnata & Sapindaceae & Climber & Unknown \\
\hline Pennisetum purpureum & Malvaceae & Herb & Least concern \\
\hline Phyllantus discoideus & Euphorbiaceae & Shrub & Unknown \\
\hline Phoenix reclinta & Araceaae & Climber & Unknown \\
\hline Picralima elliotti & Apocynacea & Tree & Unknown \\
\hline Piptadeniastrum africanum & Fabaceae & Tree & Unknown \\
\hline Placodiscus Splendidus & Spindaceae & Tree & Unknown \\
\hline Psidium guajava & Myrtaceae & Shrub & Unknown \\
\hline Tabernacmontana crassa & Apocynacea & Shrub & Unknown \\
\hline Terminalia superba & Combretaceae & Tree & Least concern \\
\hline Tetracera potatoria & Dilleniaceae & Climber & Unknown \\
\hline Triclisia patens & menispermacea & Climber & Unknown \\
\hline Trilepis pilosa & Cyperaceae & Herb & Unknown \\
\hline
\end{tabular}


Continued

\begin{tabular}{cccc}
\hline Santira trimera & Burseraceae & Shrub & Unknown \\
Scleria barteri & Cyperaceae & Herb & Unknown \\
Spondias mombin & Anacardiaceae & Tree & Least concern \\
Sporobolus dinklagei & Gramineae & Unidentified & Unknown \\
Solanum torvum & Solanaceae & Shrub & Unknown \\
Sorindeia Juglandifolia & Anacardiaceae & Shrub & Least concern \\
Sterculia tragacantha & Malvaceae & Shrub & Least concern \\
Strychnos nuxvomica & Loganiaceae & Herb & Unknown \\
Urera oblongifolia & Urticaceae & Climber & Unknown \\
Uvaria Chamae & Annonacea & Shrub & Unknown \\
Vangueriopsis discolor & Rubiaceae & Shrub & Unknown \\
Vernonia amygdalina & Compositae & Shrub & Unknown \\
Vitex cuneata & Lamiaceae & Tree & Unknown \\
Xylopia aethiopica & Annonaceae & Tree & Least concern \\
Xylopia acutiflora & Annonaceae & Shrub & Least concern \\
Xylopia quintais & Annonaceae & Tree & Unknown \\
\hline
\end{tabular}

plant species form in the different vegetative zone were Tree, Shrub, Herb, and Climber. Tree species were the most dominant life form representing $32.14 \%$ followed by shrubs 30.5 , herbs $26.8 \%$, and climbers $10 \%$. The species conservation status of the different individual plant species at Kasewe forest was determined by the IUCN list [22], and species conservation status was divided into four groups: unknown species, least concern species, vulnerable species, and data deficient species. Based on the IUCN conservation listing, fifty-seven plant species were rated as unknown at the study namely: Adenia lobata, Cercestis afzelii, Chlorophoro regia, Dialium guineense, Diodia scandens, Hibiscus physaloides, and Hippocratea Iotricha, etc. while Alchornea cordifolia, Capsicum annuum, Cassia siberrianna, Parinari excelsa, Pennisetum purpureum, Xylopia Aethiopica and Xylopia acutiflora, etc. were rated as least concern species. In contrast, Garcinia Kola and Nesogordnia Papaverifera rated as vulnerable plant species, and Mangifera indica and Manihot esculenta were also rated as data deficient species in the study area (Table 2). The most predominant families, comprising of two or more individual species considered as the same family, were Malvaceae 13.5\%, Rubiaceae 9.6\%, Fabaceae 9.6\%, Euphorbiaceae 9.6\%, Anacardiaceae 7.7\%, Verbenaceae 5.8\%, Meliaceae 5.8\%, Annonaceae 5.8\%, Zingiberaceae 3.8\%, Apocynaceae $3.8 \%$, Araceae $3.8 \%$, Musaceae $3.8 \%$ and Cyperaceae $3.8 \%$ and thirty-nine families having one species each in the study area including the following: Beilschmiedia Mannii, Ceiba pentandra, Desmodium adscendens, Impoea involucrate, Newboudia Laevis, Parinari excelsa with other families species grouped. 


\subsection{Density and Spatial Distribution of Species at the Study Area}

The density and spatial distrubtion of plant species were determined as presented in Table 3. Species with the highest densities at the study area were Chromolaena odorata, $110 \mathrm{~m}^{2}$, while Xylopia quintais, $0.1 \mathrm{~m}^{2}$, had the least density recorded per hector. Corchorus olitorius $6.6 \mathrm{~m}^{2}$, Diodia scandens $6 \mathrm{~m}^{2}$, Hymenocardia acida $5.8 \mathrm{~m}^{2}$, Lantana camara $5.5 \mathrm{~m}^{2}$, Manihot esculenta $5 \mathrm{~m}^{2}$, Mimosa paudica $5 \mathrm{~m}^{2}$, Sterculia tragacantha $5.6 \mathrm{~m}^{2}$, Nesorgordnia papaverifera $4.5 \mathrm{~m}^{2}$, and Vernonia amygdalina $4.4 \mathrm{~m}^{2}$ per hector were the common species found in the study area. The spatial distribution of plant species across the closed canopy, disturbed areas, farmland, and grassland revealed that 75 plant species were considered to be aggregated and 9 different individual species were random species within the study area, as shown in Table 3.

\subsection{Diversity of Species}

Shannon-Wiener index $(\mathrm{H})$ was used to determine the plant diversity in the different vegetative zones studied as shown in Table 4. A diversity index is the measure of species diversity in a given community. Diversity indices provide more information than merely the number of species. They serve as valuable tools that enable biologists to quantify diversity in a community and describe its numerical structure [17]. A high value of the Shannon Index $(H)$ would represent a diverse and equally distributed community, and lower values represent a less diverse community. An ecosystem with an $\mathrm{H}$ value greater than 2 has been regarded as medium to high species diversity [16] [17]. Considering the Shannon diversity index, the diversity of plant species in each plot never varies greatly although there was a significant difference noted in biodiversity between plots 2, 3, 4 in the closed canopy habitat. Meaning, the plots have common dominance plants from the closed forest canopy to the grassland. However, the diversity of plant species in plot 3 , at the closed forest canopy, recorded the highest diversity of 1.236 while plot 10 , in the grassland, was considered the lowest with 0.757 . The low biological diversity noted in some plots could be explained by the fact of the presence of human activities within the area and the dominance of Scleria barteri. Several natural causes such as soil type, rainfall trends, anthropogenic action, land-use change, encroachment, and climate change, and introduction of the invasive pest could also explain the variations in the degree of diversity between the studied plots.

\subsection{Ethnobotanical Information}

\subsubsection{Medicinal Plants Used by Respondents}

Medicinal plants mentioned by respondents and identified during the ethnobotanical study are represented in Table 5. 71 medicinal plants were named and documented that are used to treat different therapeutic ailments. Fabaceae and Euphorbiaceae were the predominant families and both representing $18.42 \%$ while Rubiaceae $15.78 \%$, Malvaceae $10.52 \%$, and Verbenaceae $7.89 \%$. This research finding supports [23] [24] who reported that most of the medicinal plants 
Table 3. Density, frequency, and species aggregation of plants sampled.

\begin{tabular}{|c|c|c|c|c|c|c|c|}
\hline Scientific name & Occur & $\mathrm{D}$ & Rde & F \% & RFr & $\% \mathrm{~F}$ & Category \\
\hline Aframomum spp & 4 & 0.4 & 0.118 & 0.1 & 0.29 & 10 & Aggregated \\
\hline Anisophyllea Laurina & 19 & 1.9 & 0.562 & 0.4 & 1.37 & 4 & Aggregated \\
\hline Alchornea cordifolia & 84 & 8.4 & 2.487 & 0.4 & 6.1 & 40 & Aggregated \\
\hline Albizia zygia & 7 & 0.7 & 0.207 & 0.1 & 0.5 & 10 & Aggregated \\
\hline Allium ascalonicum & 24 & 2.4 & 0.71 & 0.2 & 1.74 & 20 & Aggregated \\
\hline Ananas Cosmosus & 21 & 2.1 & 0.621 & 0.2 & 1.52 & 20 & Aggregated \\
\hline Adenia lobata & 16 & 1.6 & 0.473 & 0.2 & 1.16 & 20 & Aggregated \\
\hline Beilschmiedia Mannii & 14 & 1.4 & 0.414 & 0.3 & 1.01 & 30 & Aggregated \\
\hline Caraca papaya & 18 & 1.8 & 0.533 & 0.3 & 1.3 & 30 & Aggregated \\
\hline Capsicum annuum & 25 & 2.5 & 0.74 & 0.3 & 1.81 & 30 & Aggregated \\
\hline Cassia sieberiana & 8 & 0.8 & 0.236 & 0.2 & 0.58 & 20 & Aggregated \\
\hline Carapa Procera & 9 & 0.9 & 0.266 & 0.4 & 0.65 & 40 & Random \\
\hline Cercestis Afzelii & 2 & 0.2 & 0.059 & 0.2 & 0.14 & 20 & Random \\
\hline Ceiba Pentandra & 6 & 0.6 & 0.177 & 0.2 & 0.43 & 20 & Aggregated \\
\hline Chlorophoro regia & 10 & 1 & 0.296 & 0.2 & 0.72 & 20 & Aggregated \\
\hline Chromolaena Odorata & 1100 & 110 & 32.573 & 0.6 & 79.88 & 60 & Aggregated \\
\hline Citrus aurantifolia & 5 & 0.5 & 0.148 & 0.1 & 0.36 & 10 & Aggregated \\
\hline Clerodendron scandens & 9 & 0.9 & 0.266 & 0.1 & 0.65 & 10 & Aggregated \\
\hline Costus afer & 14 & 1.4 & 0.414 & 0.2 & 1.01 & 20 & Aggregated \\
\hline Corchorous olitorius & 66 & 6.6 & 1.954 & 0.3 & 4.79 & 30 & Aggregated \\
\hline Desmodium adscendens & 10 & 1 & 0.296 & 0.1 & 0.72 & 10 & Aggregated \\
\hline Dialium guineense & 7 & 0.7 & 0.207 & 0.2 & 0.5 & 20 & Aggregated \\
\hline Diodia scandens & 60 & 6 & 1.776 & 0.2 & 4.35 & 20 & Aggregated \\
\hline Diospyros heudelotii & 11 & 1.1 & 0.325 & 0.3 & 0.79 & 30 & Aggregated \\
\hline Fiscus exasperata & 4 & 0.4 & 0.118 & 0.1 & 0.29 & 10 & Aggregated \\
\hline Garcinia kola & 5 & 0.5 & 0.148 & 0.4 & 0.36 & 40 & Random \\
\hline Gmelina Arborea & 37 & 3.7 & 1.095 & 0.3 & 2.68 & 30 & Aggregated \\
\hline Gosspium spp. & 2 & 0.2 & 0.059 & 0.1 & 0.14 & 10 & Random \\
\hline Guibourtia copalifera & 122 & 12.2 & 3.612 & 0.6 & 8.85 & 60 & Aggregated \\
\hline Harungana madagascariensis & 14 & 1.4 & 0.8 & 0.414 & 0.2 & 1.01 & Aggregated \\
\hline Hibiscus esculentus & 21 & 2.1 & 0.621 & 0.5 & 1.52 & 50 & Aggregated \\
\hline Hibiscus physaloides & 8 & 0.8 & 0.236 & 0.2 & 0.58 & 20 & Aggregated \\
\hline Hippocratea iotricha & 7 & 0.7 & 0.207 & 0.3 & 0.5 & 30 & Random \\
\hline
\end{tabular}




\section{Continued}

\begin{tabular}{|c|c|c|c|c|c|c|c|}
\hline Hymenocardia acida & 58 & 5.8 & 1.717 & 0.2 & 4.21 & 20 & Aggregated \\
\hline Lantana camara & 55 & 5.5 & 1.628 & 0.1 & 3.99 & 10 & Aggregated \\
\hline Lannea & 11 & 1.1 & 0.325 & 0.4 & 0.79 & 40 & Aggregated \\
\hline Mareya micrantha & 6 & 0.6 & 0.177 & 0.3 & 0.43 & 30 & Random \\
\hline Mangifera indica & 5 & 0.5 & 0.148 & 0.3 & 0.36 & 30 & Random \\
\hline Manihot esculenta & 50 & 5 & 1.48 & 0.3 & 3.63 & 30 & Aggregated \\
\hline Mimosa pudica & 50 & 5 & 1.48 & 0.2 & 3.63 & 20 & Aggregated \\
\hline Morinda germinata & 8 & 0.8 & 0.236 & 0.2 & 0.58 & 20 & Aggregated \\
\hline Musanga cecropiodes & 10 & 1 & 0.296 & 0.1 & 0.72 & 10 & Aggregated \\
\hline Musa paradisiaca & 4 & 0.4 & 0.118 & 0.3 & 0.29 & 30 & Random \\
\hline Musa sapientum & 6 & 0.6 & 0.177 & 0.2 & 0.43 & 20 & Aggregated \\
\hline Nesogordnia papaverifera & 45 & 4.5 & 1.332 & 0.2 & 3.26 & 20 & Aggregated \\
\hline Newboudia laevis & 16 & 1.6 & 0.473 & 0.2 & 1.16 & 20 & Aggregated \\
\hline Nuclea diderrichii & 27 & 2.7 & 0.799 & 0.3 & 1.96 & 30 & Aggregated \\
\hline Nuclea latifolia & 131 & 13.1 & 3.879 & 0.5 & 9.51 & 50 & Aggregated \\
\hline Ochthocosmus africnas & 19 & 1.9 & 0.562 & 0.3 & 1.37 & 30 & Aggregated \\
\hline Ocimum viride & 5 & 0.5 & 0.148 & 0.1 & 0.36 & 10 & Aggregated \\
\hline Ouratea flava & 99 & 9.9 & 2.931 & 0.5 & 7.18 & 50 & Aggregated \\
\hline Oryza sativa & 100 & 10 & 2.961 & 0.2 & 7.26 & 20 & Aggregated \\
\hline Oxyanthus tenuis & 20 & 2 & 0.592 & 0.2 & 1.45 & 20 & Aggregated \\
\hline Panicum afzelli & 22 & 2.2 & 0.651 & 0.2 & 1.59 & 20 & Aggregated \\
\hline Parinari excelsa & 10 & 1 & 0.296 & 0.2 & 0.72 & 20 & Aggregated \\
\hline Paullinia pinnata & 3 & 0.3 & 0.088 & 0.2 & 0.21 & 20 & Random \\
\hline Pennisetum purpureum & 2 & 0.2 & 0.059 & 0.1 & 0.14 & 10 & Random \\
\hline Phyllantus discideus & 12 & 1.2 & 0.355 & 0.2 & 0.87 & 20 & Aggregated \\
\hline Phoenix reclinta & 3 & 0.3 & 0.088 & 0.1 & 0.21 & 10 & Aggregated \\
\hline Picralima elliotti & 13 & 1.3 & 0.384 & 0.2 & 0.94 & 20 & Aggregated \\
\hline Piptadeniastrum africanum & 11 & 1.1 & 0.325 & 0.2 & 0.79 & 20 & Aggregated \\
\hline Placodiscus Splendidus & 3 & 0.3 & 0.088 & 0.1 & 0.21 & 10 & Aggregated \\
\hline Psidium guajava & 4 & 0.4 & 0.118 & 0.2 & 0.29 & 20 & Random \\
\hline Tabernacmontana crassa & 32 & 3.2 & 0.947 & 0.4 & 2.32 & 40 & Aggregated \\
\hline Terminalia superba & 8 & 0.8 & 0.236 & 0.2 & 0.58 & 20 & Aggregated \\
\hline Tetracera potatoria & 11 & 1.1 & 0.325 & 0.1 & 0.79 & 10 & Aggregated \\
\hline Triclisia patens & 19 & 1.9 & 0.562 & 0.3 & 1.37 & 30 & Aggregated \\
\hline Trilepis pilosa & 10 & 1 & 0.296 & 0.1 & 0.72 & 10 & Aggregated \\
\hline
\end{tabular}




\section{Continued}

\begin{tabular}{cccccccc}
\hline Santira trimera & 7 & 0.7 & 0.207 & 0.1 & 0.5 & 10 & Aggregated \\
Scleria barteri & 430 & 43 & 12.735 & 0.4 & 31.22 & 40 & Aggregated \\
Spondias mombin & 14 & 1.4 & 0.4141 & 0.2 & 1.01 & 20 & Aggregated \\
Sporobolus dinklagei & 11 & 1.1 & 0.325 & 0.1 & 0.79 & 10 & Aggregated \\
Solanum torvum & 20 & 2 & 0.592 & 0.1 & 1.45 & 10 & Aggregated \\
Sorindeia Juglandifolia & 16 & 1.6 & 0.473 & 0.3 & 1.16 & 30 & Aggregated \\
Sterculia tragacantha & 56 & 5.6 & 1.658 & 0.2 & 4.06 & 20 & Aggregated \\
Strychnos nuxvomica & 14 & 1.4 & 0.414 & 0.3 & 1.01 & 30 & Aggregated \\
Urera oblongifolia & 20 & 2 & 0.592 & 0.1 & 1.45 & 10 & Aggregated \\
Uvaria Chamae & 14 & 1.4 & 0.414 & 0.2 & 1.01 & 20 & Aggregated \\
Vangueriopsis discolor & 34 & 3.4 & 1.006 & 0.3 & 2.46 & 30 & Aggregated \\
Vernonia amygdalina & 44 & 4.4 & 1.302 & 0.3 & 3.19 & 30 & Aggregated \\
Vitex cuneata & 15 & 1.5 & 0.444 & 0.2 & 1.08 & 20 & Aggregated \\
Xylopia Aethiopica & 4 & 0.4 & 0.118 & 0.2 & 0.29 & 20 & Aggregated \\
Xylopia acutiflora & 2 & 0.2 & 0.059 & 0.1 & 0.14 & 10 & Random \\
Xylopia quintais & 1 & 0.1 & 0.029 & 0.1 & 0.07 & 10 & Random \\
\hline
\end{tabular}

D (density), Rde (relative density), F (frequency), \% Rf (relative frequency), \% F (frequency \%).

Table 4. Diversity values by index.

\begin{tabular}{cc}
\hline Plots & Shannon H \\
\hline 1 & 0.791 \\
2 & 1.036 \\
3 & 1.236 \\
4 & 1.142 \\
$\mathbf{5}$ & 0.795 \\
$\mathbf{6}$ & 0.79 \\
$\mathbf{7}$ & 0.876 \\
$\mathbf{8}$ & 1.136 \\
$\mathbf{9}$ & 0.913 \\
10 & 0.757 \\
\hline
\end{tabular}

Table 5. Medicinal plants harvested and used by respondents from the communities.

\begin{tabular}{ccc}
\hline Local Names & Scientific names & Family names \\
\hline nGobei & Abrus precatorius & Fabaceae \\
Aligeta pepe & Aframomum melegueta & Zingiberaceae \\
\hline
\end{tabular}




\section{Continued}

nJekoi
Garlic
Kandie
Pineaple
Bush-ataya
Gbengba
Sekou-Toure
Mbelei
Pawpaw
Tambei
nGuwei
Lem
Hona-Wuli
Nyele
Kola

Lemon grass

Kolei

Am-pentakeli

nGogbei

So-Wuli

Kamaama

Bitter-kola

Yamami

Sawawa

Koboi

Blood Tick

Gonellevei

Korwei

Okara

Kagbalkantha

nDondonqui

Telei

Cassava leaf

Mangoi
Alchornea cordifolia

Allium sativum

Anisophyllea laurina

Ananas comosus

Aspalathus linearis

Cassia siberrianna

Cassia siamea

Canarium schweinfurthii

Caraca papaya

Calamus deeratus

Ceiba pantandra

Citrus aurantifolia

Clerodendron scandens

Craterispermum laurinum

Cola nitida

Cybopogen citratus

Detarium senegalense

Dichrostachy glomerata

Erythrophleum invorense

Fagana macrophylla

Fiscus exasperata

Garcina kola

Gmeline arborea

Gouania longipetala

Guibourtia copallifera

Harungana madagascariens

Hibiscus physaloide

Hibiscus sterculifolius

Hibiscus esculentus

Hymencardia lyrate

Impoea involucrata

Imerata cylindrica

Manihot esculenta

Mangifera indica
Euphorbiaceae

Alliaceae

Rhizophoracea

Bromeliaceae

Linearis

Fabaceae

Leguminosae

Burseraceae

Meliaceae

Arecaceae

Bombacaceae

Rutaceae

Verbenaceae

Rubiaceae

Sterculiaceae

Gramineae

Fabaceae

Chailletiaceae

Fabaceae

Ruaceae

Moracea

Clusiacea

Verbenaceae

Rhamnaceae

Fabaceae

Hypericaceae

Malvaceae

Malvaceae

Malvaceae

Euphorbiaceae

Convolvulaceae

Gramineae

Euphorbiaceae

Anacardiaceae 


\section{Continued}

\begin{tabular}{|c|c|c|}
\hline Badobrup & Mezoneurum benthamianum & Leguminosae \\
\hline Tolugbele & Milletia rhodantha & Fabaceae \\
\hline Sensitive/Gba-Gbema & Mimosa pudica & Fabaceae \\
\hline Moringa & Moringa oleifera & Moringaceae \\
\hline nJasui & Morinda germinata & Rubiaceae \\
\hline Banana & Musa sapientum & Musaceae \\
\hline Ka-fen fen/nGolo-Hema & Napoleona heudelotii & Lecythidacea \\
\hline Kojaagei & Nesogordinia papaverifera & Malvacea \\
\hline Pomamgbei & Newbouldia laevis & Bignoniaceae \\
\hline Bundui & Nuclea diderrichii & Rubiaceae \\
\hline Yumbuyambei & Nuclea latifolia & Rubiaceae \\
\hline Rice & Oryza sativa & Gramineae \\
\hline Tombei & Oxyanthus tenuis & Rubiaceae \\
\hline Am-fent & Paullina pinnata & Sapindaceae \\
\hline nDawei & Parinari excelsa & Chrysobalanaceae \\
\hline Tijo & Phyllanthus discoideus & Euphorbiaceae \\
\hline Kamajovondo & Premna hispidia & Verbenaceae \\
\hline Gogboi & Protemeagabaria stapfiana & Euphorbiaceae \\
\hline Guava & Psidium guajava & Myrtaceae \\
\hline Gbolei & Ricinodendron heudelotii & Euphorbiaceae \\
\hline Pondolivai & Scaparia dulcis & Plantaginaceae \\
\hline Dengbe-wuli & Scytopetalum tieghemii & Scytopetalaceae \\
\hline Mandei & Sesamum indicum & Pedaliaceae \\
\hline Gobji & Spondais mombin & Anacardiaceae \\
\hline nDopanei & Tetracera potatoria & Dilleniaceae \\
\hline Nenii & Thaumatocoecus daniellii & Marantaceae \\
\hline Foklobe & Triclisia paten & Cyperaceae \\
\hline An-thombo & Urera oblongitofolia & Urticaceae \\
\hline Negbojei & Uvaria chamae & Annonacea \\
\hline Totengi & Vengueriopsis discolor & Rubiaceae \\
\hline Kokoei/coco-yam & Xanthosoma sagitifolium & Arecaceae \\
\hline am-pos/Huwei & Xylopia aethiopica & Annonaceae \\
\hline Ginger & Zingiber officinale & Zingiberaceae \\
\hline
\end{tabular}


that were identified by the respondents belong to the Fabaceae (22.52\%). During the ethnobotanical studies, the respondents' predominately use shrubs representing $38.8 \%$, followed by trees with $30.55 \%$, while other life forms were herbs representing $16 \%$ and $11 \%$ for climbers. These plant parts are used used to treat many therapeutic ailments among the inhabitants of forest fringe communities around the Kasewe forest.

\subsubsection{Sources of Medicinal Plants}

A very high proportion of residents harvest plants from the Kasewe Forest as shown in Figure 2. The finding revealed that about $73.68 \%$ noted that they harvest plants from the primary forest for use as medicine. In comparison, secondary forest is $12.28 \%$ and $10.53 \%$ harvest plants from their respective farm closer to their communities and $1.75 \%$ from their garden. The findings from this research conforms with [25] that majority of species of medicinal plants use by forest fringe communities around the world are harvested in the wild rather than cultivated by the inhabitants. However, [8] reported that medicinal plants were collected from farmland converted from forest to cropland.

\subsubsection{Distance Covered}

The distance covered by respondents' in harvesting traditional medicine is presented in Figure 3. The majority of the respondents covered $10-20 \mathrm{~km}$ to harvest medicinal plants from different habitats while some communities are just a few

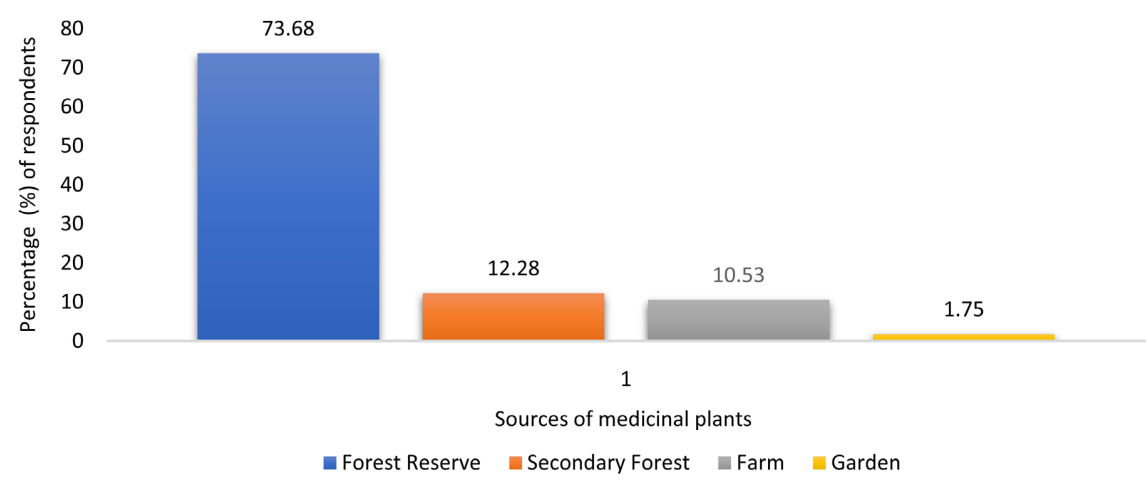

Figure 2. Sources of medicinal plants use.

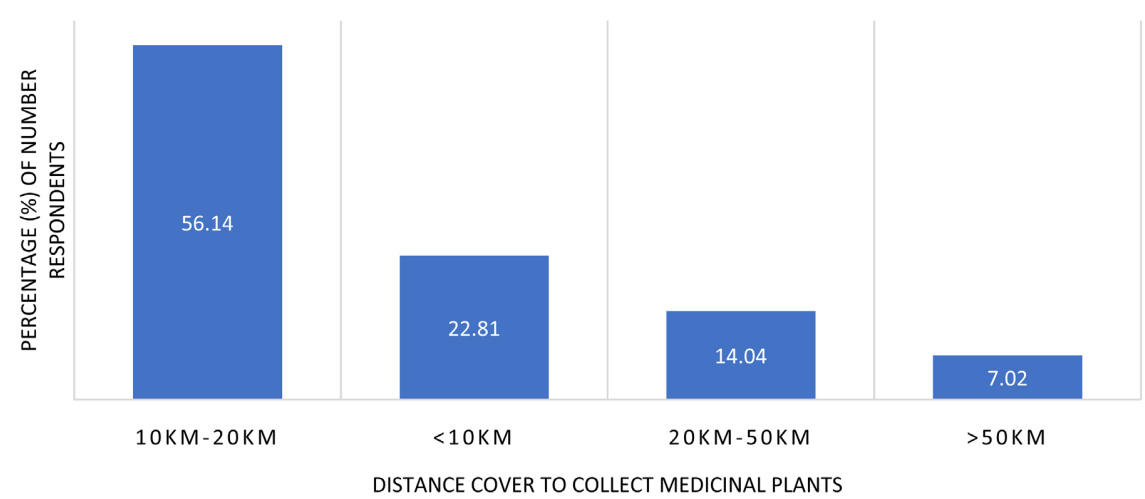

Figure 3. Distance covered to harvest medicinal plants. 
kilometers away from the forest. An implication of these findings is that the inhabitants could easily access medicinal plants because they reside around the forest. The easy accessibility to medicinal plants can promote intense use and consequently unsustainable harvesting with the possibility of overexploitation.

\subsubsection{Reasons Why People Used Medicinal Plants to Treat Therapeutic Ailments}

This study revealed that respondents used medicinal plants over modern drugs to treat therapeutic sicknesses due to numerous challenges faced in acquiring modern drugs. The lack of financial resources to purchase conventional medicine, distance of health centers, the causes of the ailments and efficacy of conventional medicine were the most reasons cited for the reliance on medicinal plants. Of views, lack of financial resources, about $50.88 \%$, was the highest while $17.54 \%$ of the respondents reported the long-distance covered to nearby health centers for treatment, and $12.28 \%$ of respondents said due to slow efficacy of conventional medicine. The finding of this study area is analogous to the view of [26] [27], that diverse cultures around the world describe the motives of using medicinal plants for the treatment of different therapeutic ailments among inhabitants of rural communities in different ways and perceptions, and also that ailment is caused by the wickedness of necromancers, wizards. Forest fringe communities around the Kasewe forest in the southern part of Sierra Leone are in remote areas that are cut off in terms of infrastructure with one health center with low economy activities. Most of inhabitants can't afford to buy conventional medicine because they are heavily involved in subsistence farming and burning of charcoal for livelihood; therefore, they instead used medicinal plants to treat their children, relatives, and friends, because nurses at the hospital prescribed drugs for them to purchase at different dispensaries centers where the hospital is situated.

\subsubsection{Medicinal Plants Used by Respondents}

Medicinal plants mentioned by respondents and identified during the ethnobotanical study on forest fringe communities around the Kasewe forest in Moyamba district are represented in Table 6. Out of the total eighth three (84) plant's species enumerated in the Kasewe, thirty-six (36) medicinal plants species mentioned by the respondents by inhabitants of forest fringe communities proximate to Kasewe forest were enumerated during the plant diversity at the Kasewe forest representing $43.3 \%$ the plant species documented at Kasewe forest. The species validified by respondents during the ethnobotanical studies predominately to be shrubs representing $38.8 \%$ followed by trees with $30.55 \%$ while other life forms were herbs representing $16 \%$ and $11 \%$ for climbers. These medicinal plants ascribed by the respondents during the ethnobotanical studies are respectively used to treat many therapeutic ailments among the inhabitants of forest fringe communities around the Kasewe forest. Majority of the respondents reported that medicinal plants collected from the forest are used for the treatment of 
Table 6. Medicinal use by communities around the kasewe forest reserve in the southeren part of Sierra Leone.

\begin{tabular}{|c|c|c|c|}
\hline Local Names & Scientific names & Family names & Therapeutic ailments \\
\hline nGobei & Abrus precatorius & Fabaceae & infertality, \\
\hline Aligeta pepe & Aframomum melegueta & Zingiberaceae & back pain, \\
\hline nJekoi & Alchornea cordifolia & Malvacea & $\begin{array}{c}\text { headache, eye problem, gonorrhea, } \\
\text { dysentry, diarrhoea, Ulcer }\end{array}$ \\
\hline Garlic & Allium sativum & Alliaceae & Impotency, Ulcer \\
\hline Kandie & Anisophyllea laurina & Rhizophoracea & Eye Problem, Dysentry \\
\hline Pineaple & Ananas comosus & Bromeliaceae & Malaria, Fever \\
\hline Bush-ataya & Aspalathus linearis & Linearis & Impotency, Fever \\
\hline Gbengba & Cassia siberrianna & Fabaceae & $\begin{array}{l}\text { stomach pain, fever, malaria, } \\
\text { back pain, diarrhoea }\end{array}$ \\
\hline Sekou-Toure & Cassia siamea & Leguminosae & Headache, Fever, Headache \\
\hline Mbelei & Canarium schweinfurthii & Burseraceae & Pile, Dysentry \\
\hline Pawpaw & Caraca papaya & Meliaceae & Fever, Dysentry, Diarrhea, Malaria \\
\hline Tambei & Calamus deeratus & Arecaceae & Cough, Menstrual Pain \\
\hline nGuwei & Ceiba pantandra & Bombacaceae & Cough, Stomach pain \\
\hline Lem & Citrus aurantifolia & Rutaceae & Cold, Fever, Diarrhoea, Fatigue \\
\hline Hona-Wuli & Clerodendron seandens & Verbenaceae & Ulcer, Menstrual Pain \\
\hline Nyele & Craterispermum laurinum & Rubiaceae & Cough, Stomach pain, Malaria \\
\hline Kola & Cola nitida & Sterculiaceae & Headache, Fever, Dysentry, Diarrhoea \\
\hline Lemon grass & Cybopogen citratus & Gramineae & Cough, Malaria, Fever, Fatigue \\
\hline Kolei & Detarium senegalense & Fabaceae & Cough \\
\hline Am-pentakeli & Dichrostachy glomerata & Chailletiaceae & Pile \\
\hline nGogbei & Erythrophleum invorense & Fabaceae & Cough \\
\hline So-Wuli & Fagana macrophylla & Ruaceae & Ulcer \\
\hline Kamaama & Fiscus exasperata & Moracea & Infertality, Eye problem \\
\hline Bitter-kola & Garcina Kola & Clusiacea & $\begin{array}{c}\text { Fever, Dysentry, Diarrhea, Impotency, } \\
\text { Malaria, Headache, Back Pain }\end{array}$ \\
\hline Yamami & Gmeline arborea & Verbenaceae & Malaria, Cough, Ulcer, Fever, Headche \\
\hline Sawawa & Gouania longipetala & Rhamnaceae & Menstrual Pain, \\
\hline Koboi & Guibourtia copallifera & Fabaceae & Cough, Headache \\
\hline Blood Tick & Harungana madagascariens & Hypericaceae & dysentery, infertality \\
\hline Gonellevei & Hibiscus physaloide & Malvaceae & Malaria, Fever \\
\hline Korwei & Hibiscus sterculifolius & Malvaceae & Pile \\
\hline Okara & Hibiscus esculentus & Malvaceae & Ulcer \\
\hline
\end{tabular}




\section{Continued}

\begin{tabular}{|c|c|c|c|}
\hline Kagbalkantha & Hymencardia lyrate & Euphorbiaceae & Dysentry \\
\hline nDondonqui & Impoea involucrata & Convolvulaceae & $\begin{array}{l}\text { Cough, Malaria, Fever, Stomach Pain, } \\
\text { Headache, Back Pain, Impotency }\end{array}$ \\
\hline Telei & Imerata cylindrica & Gramineae & Dysentrary, \\
\hline Cassava leaf & Manihot esculenta & Euphorbiaceae & Diarrhoea, Dysentary, Eye Problem, Wound \\
\hline Mangoi & Mangifera indica & Anacardiaceae & diarrhoea, fever, \\
\hline Badobrup & Mezoneurum benthamianum & Leguminosae & Stomach Pain, Ulcer, Fatigue \\
\hline Tolugbele & Milletia rhodantha & Fabaceae & Impotency \\
\hline Sensitive/Gba-Gbema & Mimosa pudica & Fabaceae & Pile, Dysentry \\
\hline Moringa & Moringa oleifera & Moringaceae & $\begin{array}{c}\text { Fever, Malaria, Stomach Pain, } \\
\text { Menstrual Pain, Diarrhoea, Headache }\end{array}$ \\
\hline nJasui & Morinda germinata & Rubiaceae & Stomach Pain, Malaria, Fever Headche \\
\hline Banana & Musa sapientum & Musaceae & Dysentry, Ulcer \\
\hline Ka-fen fen/nGolo-Hema & Napoleona heudelotii & Lecythidacea & Back pain, Stomach Pain \\
\hline Kojaagei & Nesogordinia papaverifera & Malvacea & Cough \\
\hline Pomamgbei & Newbouldia laevis & Bignoniaceae & Dysentary, Diarrhoea, Headache, Ulcer \\
\hline Bundui & Nuclea diderrichii & Rubiaceae & $\begin{array}{c}\text { Fever, Malaria, Stomach Pain, Menstrual Pain, } \\
\text { Cough, Diarrhoea, Headache, Fatigue }\end{array}$ \\
\hline Yumbuyambei & Nuclea latifolia & Rubiaceae & $\begin{array}{l}\text { Cough, Malaria, Fever, Stomach Pain, } \\
\text { Headache, eye problem, Back Pain }\end{array}$ \\
\hline Rice & Oryza sativa & Gramineae & Pile \\
\hline Tombei & Oxyanthus tenuis & Rubiaceae & Cough \\
\hline Am-fent & Paullina pinnata & Sapindaceae & Pile, Ulcer \\
\hline nDawei & Parinari excelsa & Chrysobalanaceae & $\begin{array}{l}\text { pregnancy Pain, diarrhoea, } \\
\text { dysentery, infertality }\end{array}$ \\
\hline Tijo & Phyllanthus discoideus & Euphorbiaceae & Headache \\
\hline Kamajovondo & Premna hispidia & Verbenaceae & Back pain, Dysentry, Malaria \\
\hline Gogboi & Protemeagabaria stapfiana & Euphorbiaceae & Headache \\
\hline Guava & Psidium guajava & Myrtaceae & $\begin{array}{l}\text { Dysenatary, Diarrhoea, Cough, } \\
\text { Stomach Pain, Ulcers }\end{array}$ \\
\hline Gbolei & Ricinodendron heudelotii & Euphorbiaceae & Cough \\
\hline Pondolivai & Scaparia dulcis & Plantaginaceae & Back pain, Fatigue \\
\hline Dengbe-wuli & Scytopetalum tieghemii & Scytopetalaceae & Diarrhoea, Dysentary, \\
\hline Mandei & Sesamum indicum & Pedaliaceae & Eye Problem \\
\hline Gobji & Spondais mombin & Anacardiaceae & Headache \\
\hline nDopanei & Tetracera potatoria & Dilleniaceae & Cough \\
\hline Nenii & Thaumatocoecus daniellii & Marantaceae & Menstrual Pain, \\
\hline
\end{tabular}




\begin{tabular}{cccc}
\hline Foklobe & Triclisia paten & Cyperaceae & $\begin{array}{c}\text { Cough, Back pain, Stomach Pain, } \\
\text { Fatigue, Malaria }\end{array}$ \\
An-thombo & Urera oblongitofolia & Urticaceae & $\begin{array}{c}\text { Back Pain, Dysentry, Stomach Pain, } \\
\text { Malaria, Headache }\end{array}$ \\
Negbojei & Uvaria chamae & Annonacea & Dysentry \\
Totengi & Vengueriopsis discolor & Rubiaceae & Dysentry, \\
Kokoei/coco-yam & Xanthosoma sagitifolium & Arecaceae & Malaria \\
am-pos/Huwei & Xylopia aethiopica & Annonaceae & Cough, Menstrual Pain \\
Ginger & Zingiber officinale & Zingiberaceae & Cough, Fever, Malaria, Headche, Fatigue
\end{tabular}

malaria, because nowadays in the country, and the world in the $21^{\text {st }}$ century, malaria is a problem to human health and has caused more death in the developing countries.

\subsubsection{Sources of Traditional Medicine Knowledge}

Knowledge of medicinal plants comes from different sources. The respondents' sources of traditional medicine knowledge are presented in Table 7. Oral communication was the most source of traditional medicine knowledge. Only a few people acquired their knowledge from dreams, which represents $3.5 \%$ of the respondents. This makes it difficult for information to be retained and is often lost over the years since it not documented. The study revealed that $98.25 \%$ of the respondents with medicinal plants' knowledge for treating illnesses are older people while $1.75 \%$ are middle age. This means elders are more knowledgeable about medicinal plants harvest and use to treat different therapeutic ailments. Many other researches have reported similar findings that elderly people in rural communities worldwide are more knowledgeable on medicinal plants' uses in treating various therapeutic diseases [28] [29] [30] [31].

[30] reported that in Eastern Desert Region of Egypt, many knowledgeable elders provide the other community members with traditional knowledge on medicinal plants' uses to treat different illnesses among members of the rural community. Sharing traditional information in treating different sicknesses using medicinal plants in rural communities is essential because, in isolated communities, knowledge disappears gradually since it is orally pass on by indigenous old people to the younger generation. In addition, medicinal plant knowledge may be more difficult to acquire than other types of ethno-botanical knowledge, such as edible plants, firewood, or building materials, because of the more complex nature of medicinal plant remedies and the lower frequency of learning events.

\subsubsection{Level of Quantity Harvested}

Figure 4 presents the level of harvesting medicinal plants from the forest. The majority of the respondents' harvest plant parts moderately, representing $54 \%$, 
whereas 39\% harvest low and 7\% harvest a lot. This finding indicates that most of the respondents don't want the medicinal Plant to be eroded from the forest because some medicinal plants in the study area are scares to harvest from different habitats around the forest reserve.

\subsubsection{Method of Harvesting Plant Parts}

The respondent used different methods for harvesting medicinal plants from the forest and other habitats around the Kasewe forest as shown in Figure 5. The most method used is bark slashing while very limited proportion use tree falling especially for Xylopia aethiopica. The method most used by inhabitants is unsustainable and it pose serious conservation threats. According to [32], "method

Table 7. Source of knowledge on traditional herbs.

\begin{tabular}{cc}
\hline Source of knowledge & Percentage (\%) \\
\hline Learned from parents & 66.67 \\
Learned from friends & 26.32 \\
Learned from herbalist & 1.75 \\
Learned from dreams & 3.5 \\
\hline
\end{tabular}

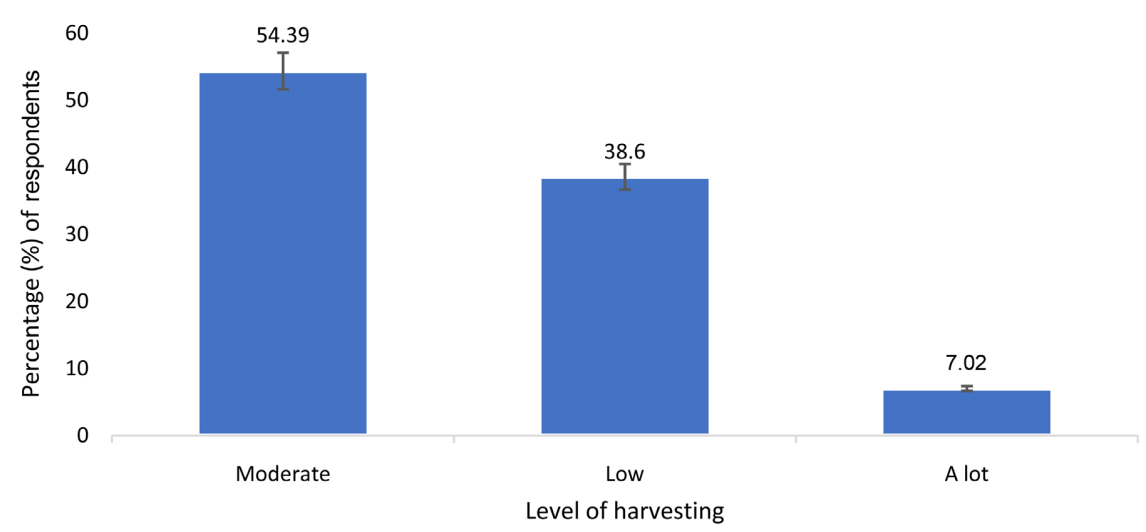

Figure 4. Level of plant parts harvested.

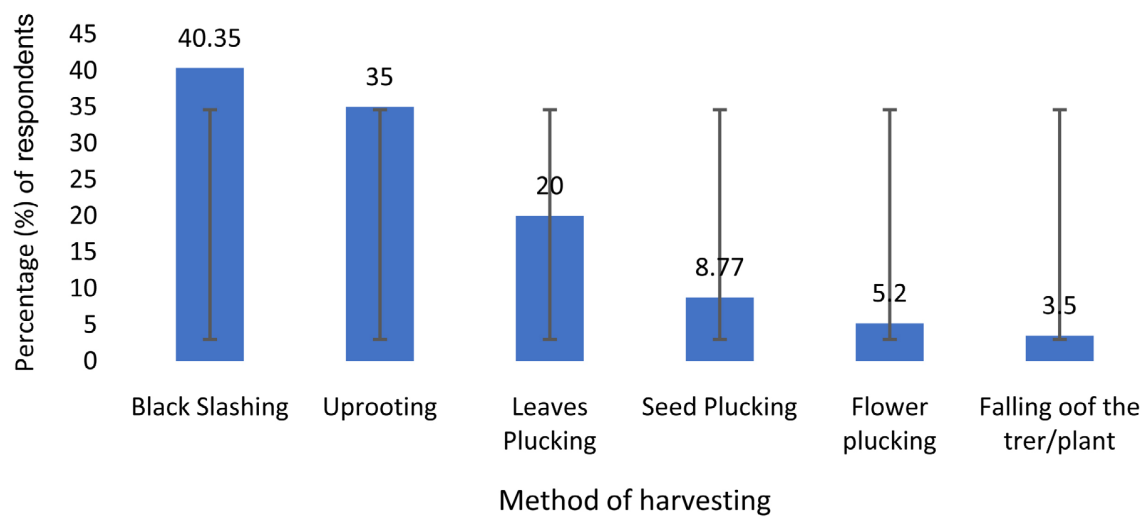

Figure 5. Method of harvesting plant species. 


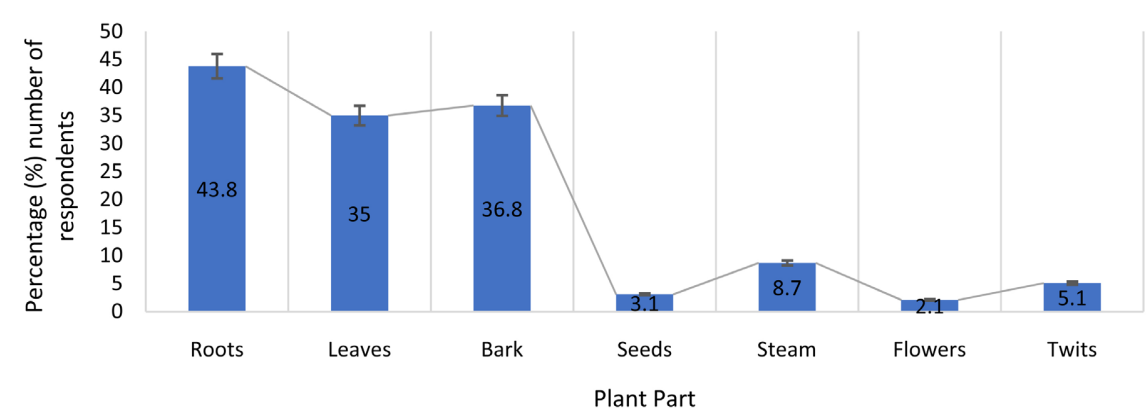

Figure 6. Diversity of plant parts used in traditional medicine in the communities.

of harvesting is related to the plant parts' predilection for the preparation of medicines to treat different therapeutic ailments." Harvesting methods can impact conservation because some types of harvesting are more sustainable than others. For example, harvesting leaves may be more bearable because of the rejuvenation of leaves in contrast with the bark, which may not be fully regenerated over a long period due to the slashing. Harvesting bark and tree species' roots may pose a threat to sustainable harvesting [25]. The high demand of medicinal plants in developing countries has led to indiscriminate harvesting [4]. This has resulted in many plant species becoming extinct and some endangered [7] [29] [31] [32].

\subsubsection{Measure of Plant Parts Used in Traditional Medicine in the Communities}

The measure of plant parts used to treat ailment is presented in Figure 6. There was diversity in the plant part use to treat ailments. The predominant plant part used in the study area to treat therapeutic ailments was the Plant's root (43.8\%) while $36.8 \%$ use the bark, and 35\% use leaves. According to [25], diverse parts of plants from bark root, leaves, and stems, flowers, seeds, and twit are used for the preparation of medicinal plants for the treatment of different therapeutic ailments. $43.8 \%$, scientific research in the past has facilitated the discovery of many phytochemicals from plant roots, some of which are now in clinical use, and many others are in numerous stages of preclinical and clinical studies. The enormous diversity of plants on the planet gives an auspicious expedition ahead in the quest for knowledge and cure for many diseases. The part of the Plant used and the growth and reproductive characteristics of the Plant will have significant implications in the timing for harvest and its susceptibility to overexploitation [6].

\section{Conclusion and Recommendation}

The Kasewe forest in the southern part of Sierra Leone is one of the biodiversity hotspots in the country and it has different tree species of ecological and economical value to the inhabitants of communities. Documentation of plant diversity and the human uses of important medicinal plants are counted viable to conservation and sustainable use of these flora species for the future generations 
in Sierra Leone. The total number of individual plant stems counted 3377, comprising 84 individual species belonging to 53 families from different vegetative zones. The most dominant family at the Kasewe Forest was Malvaceae and the species with the highest density was Chromolaena odorata $110 \mathrm{~m}^{2}$, and Xylopia quintais was the least density of species recorded at the study area representing $0.1 \mathrm{~m}^{2}$ per hector. The diversity among species is low. The closed or dense forest canopy recorded the highest diversity of species with an index of 1.236 and farmland 0.757. A total of seventy-one (71) medicinal plant were recorded during the study period used by the local people to treat different therapeutic ailments. The root, stem and bark are most use parts for treating ailments. However, the Kasewe Forest is under pressure from anthropogenic activities, such as medicinal plant harvesting, agriculture, poaching and settlement. These processes are affecting the ecological and biological components and ecosystem services of the forest making it prone to degradation. Majority of the inhabitants depend on medicinal plants from the forest to treat different ailment with very limited alternative. Although there is abundance of medicinal plants, the method of harvest and frequency of use pose a serious risk of extinction. Being a biodiversity hotspot and a home to prominent medicinal plants, there is need for conservation actions. Medicinal plants for the treatment of common illness in isolated communities located around forested region in Sierra Leone will continue to play a significant role in the local health care system because of different traditional believes and the lack of access to conventional medical care.

It is recommended that the government should implement medicinal plant conservation strategy and invest in conservation, standardization of measurements and hygiene of the medicinal plants preparation and use, documentation of traditional medicine knowledge, education, research and value chain market. Numerous medicines have been derived from the knowledge of tropical forest people. Therefore, there is the need to conserve medicinal plants and protect threatened species by introducing systematic cultivation of medicinal plants necessitated by continuous demand. Government should also encourage local communities to cultivate the medicinal plants through in-situ and ex-situ management approaches and organized programs for traditional herbalist.

Further inventory of plants should be carried out in the area in order to eventually capture all species present there. In doing so, new or endangered species may be identified which might be relevant to conservation.

\section{Acknowledgements}

Our deepest gratitude goes to God for his protection and guidance. We also extend our profound thanks and appreciation to the chiefs, elders and respondents for their cooperation, and also Mr Momoh Sesay, a botanist at the National herbarium, for his enormous contribution to this research project.

\section{Funding}

There was no funding provided by any organization. However, funding for this 
study was from the authors.

\section{Conflicts of Interest}

The authors declare no conflicts of interest regarding the publication of this paper.

\section{References}

[1] Secretariat of the Convention on Biological Diversity (2009) The Convention on Biological Diversity Plant Conservation Report: A Review of Progress in Implementing the Global Strategy of Plant Conservation (GSPC). Secretariat of the Convention on Biological Diversity, Montreal.

[2] MacFoy, C. (2014) Medicinal Plants and Traditional Medicine in Sierra Leone. iUniverse LLC, Bloomington.

[3] Kabba, M. (2017) Ethno-Botanical Survey of Medicinal Plants Used to Treat Local People around Kasewe Forest Reserve, Moyamba District, Sierra Leone. Unpublished master science, Njala University, Bo.

[4] Augustino, S. and Gillah, P.R. (2005) Medicinal Plants in Urban Districts of Tanzania: Plants, Gender Roles and Sustainable Use. International Forestry Review, 7, 44-58. https://doi.org/10.1505/ifor.7.1.44.64157

[5] Bekalo, T.H., Woodmatas, S.D. and Woldemariam, Z.A. (2009) An Ethnobotanical Study of Medicinal Plants Used by Local People in the Lowlands of Konta Special Woreda, Southern Nations, Nationalities and Peoples Regional State, Ethiopia. Journal of Ethnobiology and Ethnomedicine, 5, Article No. 26. https://doi.org/10.1186/1746-4269-5-26

[6] Brown, K. (1992) Medicinal Plants, Indigenous Medicine and Conservation of Biodiversity I Ghana. Working Paper GEC 92-36, Centre for Social and Economic Research on the Global Environment, Norwich.

[7] Chandra, P.K. (2000) Status and Conservation of Rare and Endangered Medicinal Plants in the Indian Trans Himalaya. Biological Conservation, 93, 371-379. https://doi.org/10.1016/S0006-3207(99)00128-7

[8] Ewudzie, E.E. (2013) Inventory and Ethnobotanical Studies of Medicinal Plants in the Asantemanso Sacred Grove, Ashanti Region, Ghana. Master's Thesis, Kwame Nkrumah University, Kabwe.

[9] Estomba, D., Ladio, A. and Lozada, M. (2006) Medicinal Wild Plant Knowledge and Gathering Patterns in a Mapuche Community from North-Western Patagonia. Journal of Ethnopharmacology, 103, 109-119. https://doi.org/10.1016/j.jep.2005.07.015

[10] Ewusie, J.Y. (1980) Elements of Tropical Ecology: With Reference to the African, Asian, Pacific, and New World Tropics. Heinemann Educational Books, London.

[11] Food and Agriculture of the Organization (1997) Medicinal Plants for Forest Conservation and Heath cAre. Vol. 92, Edited by Gerard Bodeker, K.K.S. Bhat of Green College, University of Oxford, Jeffrey Burley Oxford Forestry Institute University of Oxford, UK.

[12] United Nation Environment Programme (2008) World Database on Protected Areas: Site Information. World Conservation Monitoring Centre, Cambridge.

[13] Martin, G. (1995) Ethnobotany: A Methods Manual. WWF International, UNESCO and Royal Botanic Gardens, Kew/Chapman and Hall, London.

[14] Fabricant, D.S. and Farnsworth, N.R. (2001) The Value of Plants Used in Tradition- 
al Medicine for Drug Discovery. Environ Health Perspect, 109, 69-75. https://doi.org/10.1289/ehp.01109s169

[15] Curtis, J.T. and McIntosh, R.P. (1950) The Interrelations of Certain Analytic and Synthetic Phytosociological Characters. Ecology, 31, 434-455. https://doi.org/10.2307/1931497

[16] Kershaw, Kenneth A. (1973) Quantitative and Dynamic Plant Ecology. Edward Arnold, London.

[17] Shannon, C.E. and Weaver, W. (1949) The Mathematical Theory of CommunicationUniversity of Illinois Press, Urbana.

[18] Pieroni, A., Nedelcheva, A. and Dogan, Y. (2015) Local Knowledge of Medicinal Plants and Wild Food Plants among Tatars and Romanians in Dobruja (South-East Romania). Genetic Resources and Crop Evolution, 62, 605-620. https://doi.org/10.1007/s10722-014-0185-3

[19] Blanco, J. and Carrière, S.M. (2016) Sharing Local Ecological Knowledge as a Human Adaptation Strategy to Arid Environments: Evidence from an Ethnobotany Survey in Morocco. Journal of Arid Environments, 127, 30-43. https://doi.org/10.1016/j.jaridenv.2015.10.021

[20] Kankara, S.S., Ibrahim, M.H., Mustafa, M. and Go, R. (2015) Ethnobotanical Survey of Medicinal Plants Used for Traditional Maternalhealthcare in Katsina State, Nigeria. South African Journal of Botany, 97, 165-175. https://doi.org/10.1016/j.sajb.2015.01.007

[21] Tugume, P., Kakudidi, E.K., Buyinza, M., Namaalwa, J., Kamatenesi, M., Mucunguzi, P., et al. (2016) Ethnobotanical Survey of Medicinal Plant Species Used by Communities around Mabira Central Forest Reserve, Uganda. Journal of Ethnobiology and Ethnomedicine, 12, Article No. 5. https://doi.org/10.1186/s13002-015-0077-4

[22] Motaleb, M.A. (2010) Approaches to Conservation of Medicinal Plants and Traditional Knowledge: A Focus on the Chittagong Hill Tracts. IUCN (International Union for Conservation of Nature). Bangladesh Country Office, Dhaka, viii+30.

[23] Kigen, G.K., Ronoh, H.K., Kipkore, W.K. and Rotich, J.K. (2013) Current Trends of Traditional Herbal Medicine Practice in Kenya: A Review. African Journal of Pharmacology and Therapeutics, 2, 32-37.

[24] Supiandi, M.I., Mahanal, S., Zubaidah, S., Julung, H. and Ege, B. (2019) Ethnobotany of Traditional Medicinal Plants Used by Dayak Desa Community in Sintang, West Kalimantan, Indonesia. Biodiversitas, 20, 1264-1270. https://doi.org/10.13057/biodiv/d200516

[25] Hamilton, A.C. (Ed.) (2008) Medicinal Plants in Conservation and Development: Case Studies and Lessons Learnt. Plant Life International, Salisbury.

[26] Kennedy, E. (2011) Herbalism, Spirituality and Treating Diseases. Timber Press, Portland.

[27] Ver Beek, K.A. (2010) Spirituality: A Development Taboo. Journal of Development in Practice, 10, 31-43. https://doi.org/10.1080/09614520052484

[28] Kunwar, R.M., Acharya, R.P. and Bussmann, R.W. (2010) Medicinal Plants in Nepal West Himalaya: Status, Trade, Use and Community Management. Journal of Ethnobotany Research and Applications, 3, 89-106.

[29] Ranjith, M., Russell-Smith, J. and Karunaratne, N.S. (2006) Rapid Inventory of Wild Medicinal Plant Populations in Sri Lanka. Biological Conservation, 132, 22-32. https://doi.org/10.1016/j.biocon.2006.03.009

[30] Mahmoud, T. and Gairola, S. (2013) Traditional Knowledge and Use of Medicinal 
Plants in the Eastern Desert of Egypt: A Case Study from Wadi El-Gemal National Park. Journal of Medicinal Plants Studies, 1, 10-17. http://www.plantsjournal.com

[31] Tklehaymanot, T. and Giday, M. (2007) Ethnobotanical Study of Medicinal Plants Used by People in Zegie Peninsula, Northwestern Ethiopia. Journal of Ethnobiology and Ethnomedicine, 3, Article No. 12. https://doi.org/10.1186/1746-4269-3-12

[32] Caldwell, K.I. (2007) Assigning Medicinal Plant Value and Estimating Traditional Environmental Knowledge in Ghana, Africa Using Ethnobotanical Measures. M.Sc. Thesis, North Carolina State University, Raleigh. 